\title{
Photometry of SN 2002bo with template image subtraction
}

\author{
Gy. M. Szabó ${ }^{1,3}$, K. Sárneczky ${ }^{2,3}$, J. Vinkó ${ }^{2,3}$, B. Csák ${ }^{1,3}$, Sz. Mészáros ${ }^{2,3}$, P. Székely ${ }^{1,3}$, and Zs. Bebesi ${ }^{3}$ \\ 1 Department of Experimental Physics \& Astronomical Observatory, University of Szeged, 6720 Szeged, Dóm tér 9., Hungary \\ 2 Department of Optics \& Quantum Electronics \& Astronomical Observatory, University of Szeged, POB 406, 6701 Szeged, \\ Hungary \\ ${ }^{3}$ Guest Observer at Piszkéstető Station, Konkoly Observatory, Hungary
}

Received 3 June 2003 / Accepted 24 June 2003

\begin{abstract}
VRI photometry of the type Ia supernova 2002bo is presented. This SN exploded in a dusty region of the host galaxy NGC 3190, thus, subtraction of a template frame was necessary to obtain reliable photometry. We used a template frame of NGC 3190 taken during the course of our galaxy imaging project, fortunately, just a few days before SN 2002bo was discovered. The aim of this project is to collect template frames of nearby galaxies that are potential hosts of bright SNe. Subtraction of pre-SN images helped us to exclude the background light contamination of the host galaxy. The maximum occurred at JD 2452346 , with maximal $V$ brightness of 13. 58. MLCS analysis led to $T_{0}(B)=$ JD $2452346.1 \pm 0.8$ (fiducial $B$ maximum), $E(B-V),=0.24 \pm 0.02, \mu_{0}=32.46 \pm 0.06, \Delta=-0.14 \pm 0.04 . E(B-V)=0.24(2)$ indicates a significant extinction in the host galaxy as the galactic reddening is negligible toward NGC 3190. The accepted value of $\Delta$ indicates that SN 2002bo was a slightly overluminous SN by about 0.14 relative to fiducial SN type Ia. The distance turned out to be $31.0 \pm 3 \mathrm{Mpc}$. In addition, the heavily obscured SN 2002cv was also detected on the I frame taken on JD 2452434 (June 8, 2002), and a variable star is found in the field, very close to the host galaxy.
\end{abstract}

Key words. supernovae: individual: SN 2002bo, SN 2002cv - galaxies: fundamental parameters - stars: variables

\section{Introduction}

The bright supernova SN 2002bo in NGC 3190 (belonging to the Leo III group LGG 194) was spectroscopically discovered by Kawakita et al. (2002) with GAO 0.65 telescope on March 9.6 UT. A low resolution spectrum (ranged $380-750 \mathrm{~nm}$ ) showed a highly reddened Ia type supernova, about two weeks before maximum light. The expansion velocity was measured as $19000 \mathrm{~km} \mathrm{~s}^{-1}$. Another spectrum (range 400-780 nm, resolution $2.5 \mathrm{~nm}$ ) obtained on March 10.04 UT with the Asiago 1.82 telescope, showed strong P-Cygni profiles of Si II 635 and $596 \mathrm{~nm}$. The velocity measured from Si II absorption was $17700 \mathrm{~km} \mathrm{~s}^{-1}$ (Benetti et al. 2002). No object brighter than 19.5 was found at the position of the SN on CCD frames collected on Mar. 4.0 UT (Sárneczky \& Bebesi 2002).

Matheson et al. (2002) reported an expansion velocity of $17900 \mathrm{~km} \mathrm{~s}^{-1}$ on March 10.25 UT. Strong, narrow Na D absorption at the velocity of the host galaxy showed that there is probably higher reddening caused by the interstellar matter inside NGC 3190. The galactic reddening toward NGC 3190 is only $E(B-V)=00^{\mathrm{m}} 025$, according to Schlegel et al. (1998). Several titanium absorption lines in the spectrum (reported by Matheson et al. 2002) suggested that this may be a subluminous event similar to SN 1999by.

Send offprint requests to: Gy. M. Szabó, e-mail: szgy@mcse.hu
The possible underluminosity is confirmed by Chornock et al. (2002) from unusually strong Si II $(585 \mathrm{~nm})$ absorption. With the Shane 3-m reflector they measured an expansion velocity of about $16000 \mathrm{~km} \mathrm{~s}^{-1}$ from the Si II $(635.5 \mathrm{~nm})$ line on March 11.0 UT.

The host galaxy NGC 3190 (a type SA(s)a LINER) has an integrated $B$ magnitude $11 \mathrm{~m}$. 31 corrected for galactic absorption. Its radial velocity in the optical band is $+1271 \pm 14 \mathrm{~km} \mathrm{~s}^{-1}$, which corresponds to a kinematical distance modulus $\mu=$ -31 m. 31 assuming $H_{0}=65 \mathrm{~km} \mathrm{~s}^{-1} \mathrm{Mpc}^{-1}$. The $D_{25}$ diameter is 4.3 arcsec, $\epsilon_{25}$ ellipticity is 0.58 with position angle 125 . The 3dimensional central velocity dispersion is $\sigma_{\mathrm{v}}=169 \pm 11 \mathrm{~km} \mathrm{~s}^{-1}$ according to Héraudeau \& Simien (1998). SN 2002bo appeared on the edge of a dust lane in NGC 3190 (Fig. 1), and reached a maximum visual brightness of about 13. 6 by mid March 2002 .

Distance modulus to NGC 3190 is not concordant in the literature. According to Héraudeau \& Simien (1998), the dynamical distance modulus is $\mu_{0}=31.231\left(H_{0}=75 \mathrm{~km} \mathrm{~s}^{-1} \mathrm{Mpc}^{-1}\right)$, while based on the Virgocentric-fall corrected radial velocity, van Driel et al. (2001) gives only $17 \mathrm{Mpc}$ distance, $\mu_{0}=31$. 15 $\left(H_{0}=65 \mathrm{~km} \mathrm{~s}^{-1} \mathrm{Mpc}^{-1}\right)$. However, the average radial velocity of the Leo III group is about $1400 \mathrm{~km} \mathrm{~s}^{-1}$, thus, the distance to its members may be more than discussed above. Based on the Tully-Fisher relation, de Vaucouleurs (1991) gives $\mu_{\mathrm{TF}}=31 \mathrm{~m} .61$. Most recently, the surface brightness fluctuation method led to a significantly higher distance modulus of 


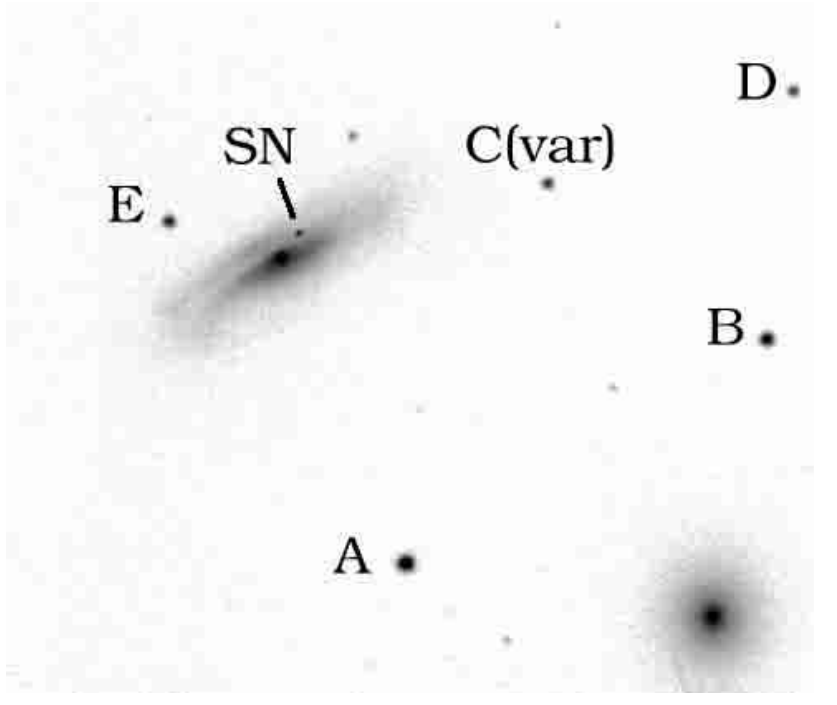

Fig. 1. SN 2002bo in NGC 3190 with the comparison stars and field variable C.

$\mu_{\mathrm{SBF}}=32.38 \pm 0.15$, indicating $30 \mathrm{Mpc}$ distance to NGC 3190 (Tonry et al. 2001).

\section{Observations and data reduction}

We have observed SN 2002 bo on 11 nights at three observing sites. Six points before light maximum and a post-maximum observation in June were measured with the 60/90/180 Schmidt-telescope of Konkoly Observatory equipped with a Photometrics AT200 CCD (1'.00 scale, RDN = 14, Vinkó et al. 2003). This telescope was also used for our host-galaxy imaging project which aims at collecting $B V R I$ frames of the closest galaxies to be used as templates for photometry of future SNe.

Most of the points were measured using the $28 \mathrm{~cm}$ SchmidtCassegrain telescope of Szeged Observatory equipped with an SBIG ST7E CCD. This $765 \times 510$ camera gives $11^{\prime \prime} 0 /$ pixel resolution, the pixels are rectangular. Despite the unfavorable location of this telescope (in the very center of the city of Szeged), $V, R$ and $I$ observations could be made with satisfactory accuracy using integrated expositions of $10-20 \times 1$ min images. All the observations between JD 2452350 and JD 2452389 were made with this equipment.

The transformation equations for Szeged Observatory and Konkoly Observatory, respectively, were applied as follows.

$$
\begin{aligned}
& V=v-0.21(8) \cdot(V-R)+\kappa_{V} \\
& (V-R)=0.83(2) \cdot(v-r)+\kappa_{V-R} \\
& (V-I)=0.88(9) \cdot(v-i)+\kappa_{V-I} \\
& V=v+0.09(3) \cdot(V-R)+\kappa_{V} \\
& (V-R)=0.98(4) \cdot(v-r)+\kappa_{V-R} \\
& (V-I)=1.13(2) \cdot(v-i)+\kappa_{V-I} .
\end{aligned}
$$

Local standards were measured at Konkoly Observatory on June 8th using standard fields PG 1633+09 and PG 1323-086 of Landolt (1992). Magnitudes and errors accepted for comparison stars are presented in Table 1 (see Fig. 1 for the designations of the stars). Uncertainties are computed by quadratic
Table 1. GSC-numbers, magnitudes and colors of local standards.

\begin{tabular}{lllll}
\hline \hline Star & GSC number & $V$ & $V-R$ & $R-I$ \\
\hline A & 1425496 & $12.28(4)$ & $0.29(3)$ & $0.26(8)$ \\
B & 142542 & $13.57(4)$ & $0.45(3)$ & $0.50(2)$ \\
D & 1425713 & $15.31(4)$ & $0.70(3)$ & $0.60(3)$ \\
E & 1425629 & $14.27(4)$ & $0.44(3)$ & $0.45(2)$ \\
\hline
\end{tabular}

summation of errors in individual measurements and the transformation coefficient errors. Star C (GSC 1425 35) varies (suspected eclipsing variable), so it was rejected from the comparison set.

SN 2002bo appeared close to the center of the host galaxy NGC 3190, so the brightness contribution of NGC 3190 must be taken into account in the reduction. The dust lane of this edge-on spiral also passes near the position of the supernova, causing a quite inhomogeneous background. In similar cases, the observer usually has to wait at least a year after the explosion, when the fireball diminishes enough to allow imaging the background brightness contribution of the host galaxy.

\subsection{Subtraction of the template}

In order to get acceptable photometry, the subtraction of a template image of NGC 3190 was necessary. Fortunately, this image was taken on March 4.0 UT, 2002 during the course of our galaxy imaging project. The aim of this image subtraction was to smooth out the background of the SN, so that the scraggy surface of NGC 3190 does not disturb the reduction. For the proper subtraction, $\mathrm{SN}$ - and template images must have the same geometry, and the same flux scale above the zeroed background. Thus, the template and $\mathrm{SN}$ frames were registered and rescaled before the template subtraction.

The geometrical transformations of the template frames consisted of a stretch and a rotation, so higher-order distortions caused by the image curvature were neglected. We used the imexamine task in IRAF $^{1}$ to make accurate astrometry of the comparison stars and the SN. The linear geometrical transformations were calculated by the geotran and imarith tasks. The fixed point of the transformations was the supernova itself, so higher-order geometrical distortions were almost negligible in the close surroundings.

As a second step, the backgrounds were zeroed on each image. The subtracted sky values were measured manually on the individual images. Fluxes of comparison stars were calculated by imexamine. The intensities of the $\mathrm{SN}$ image were rescaled so that the total fluxes (the summarized ADU values) of comparison stars A, B, D, E were the same as on the template frame.

It should be noted that the filter characteristics on Konkoly and Szeged sites are quite different. One could therefore think that the subtraction of the nominally adequate images could result in considerable residuals, as the two filters are centered on slightly different bands. Fortunatelly, even in this case the subtraction offers much better circumstances for reduction, as

\footnotetext{
1 IRAF is distributed by NOAO which is operated by the Association of Universities for Research in Astronomy (AURA) Inc. under cooperative agreement with the National Science Foundation.
} 
the overall spectrum of the host galaxy (disk region) is quite smooth. After rescaling the fluxes of the SN image, we simply subtract "something similar" to the host galaxy but without the SN. Perfect subtraction should therefore result in a clear image where nothing but the supernova is visible. Its flux is scaled onto the template system, but this does not distort the photometry.

After the template subtraction the host galaxy together with the foreground stars should vanish completely. In practice, however, the PSF slightly varies from image to image, causing varying structures and weights of the profile veils. Therefore, the PSF of the observed image was measured near the SN, and the template image was blurred with a Gaussian in order to have approximately the same PSF as the SN frames.

The result is presented in Fig. 2 where the observed and the residual images are compared. Fainter stars have vanished completely, though the higher-order component of the veils of the brighter stars remained on the frame. Though the galaxy is not perfectly subtracted, the background around the $\mathrm{SN}$ is much smoother and it could be removed much better during the photometry.

The final step was the application of a standard aperture photometry. The flux of the comparison stars were measured on the observed frames after geometric transformations and intensity scaling, but before template subtraction. The SN was measured after the subtraction. Nominal errors of the relative photometry were calculated by $I R A F$, analysing the scatter of the background near the SN and the detected flux of the SN itself.

The random errors of the differential photometry were estimated as $\sigma_{\text {rnd }}^{2}=\sigma_{\mathrm{p}}^{2}+\sigma_{\mathrm{c}}^{2}+\sigma_{\mathrm{b}}^{2}$, where $\sigma_{\mathrm{p}}$ is the error of the photometry caused by the photon noise, $\sigma_{\mathrm{c}}$ is caused by the color-dependent part of the transformations (e.g. $\Delta \epsilon \cdot(V-R)+$ $\Delta(V-R) \cdot \epsilon)$ ), and $\sigma_{\mathrm{b}}$ is the error caused by the template subtraction, namely the resultant inhomogeneities in the residual background, typically $2-6 \%$, depending on the quality of the observed images ${ }^{2}$.

Systematic errors of the standard photometry come from the measurement of the local standard stars. Those were estimated as $\sigma_{V}=0$. $04, \sigma_{R}=0.03, \sigma_{i}=0.04$. These systematic errors do not influence the shape of the light curve or the $\Delta$ and $T$ parameters in the MLCS-modelling. However, they are present the distance modulus and the extinction. Therefore we added $\sigma_{\text {sys }} \approx 0$. 04 to the fit errors of $\mu_{0}$ and $E(B-V)$.

\section{Results}

The calibrated standard magnitudes of SN 2002bo are listed in Table 2. The estimated errors of each point are given in parentheses. The VRI light curves were analyzed by the Multi-Color Light Curve Shape (MLCS) methods of Riess et al. (1996, 1998).

The timescale of the observations was divided by $1+z=$ 1.0042 to remove the effect of time dilation. The fitting was

\footnotetext{
${ }^{2}$ In our experience, the residual sky never exceeds $5 \%$ of the value of the originally observed sky. So we estimated $\sigma_{\mathrm{b}}(\%) \approx \frac{\text { SKY.0.05 }}{\text { FLUX }}$, where SKY and FLUX refer to the sky value inside the aperture and the measured brightness of the $\mathrm{SN}$, respectively.
}

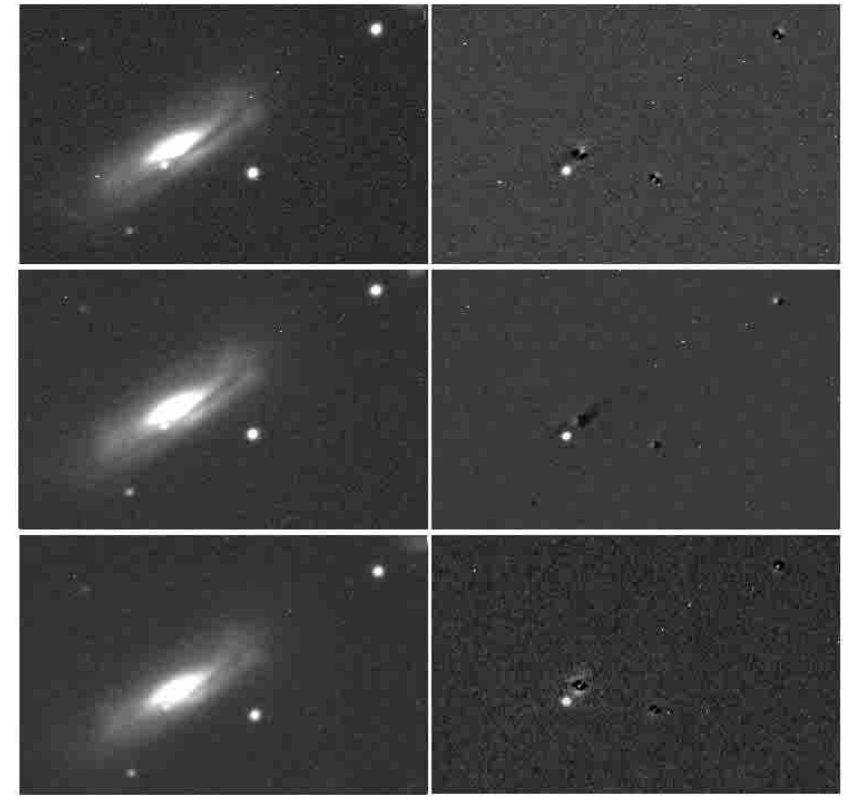

Fig. 2. Observed $V, R$ and $I$ images of SN 2002bo on 16th March (left panel), and the residual images after template subtraction (right panel).

Table 2. Observation time (JD-2 450000 ) and standard photometry of SN 2002bo. Telescope codes: A: 60/90/180 cm Schmidt, Konkoly Obs., B: $28 \mathrm{~cm}$ Schmidt-Cassegrain, Szeged Obs. The errors in parentheses refer to the accuracy of the relative photometry; see text for the systematic errors in different bands.

\begin{tabular}{lllll}
\hline \hline Time & Tel. & $V$ & $R$ & $I$ \\
\hline 2344.285 & A & $15.729(0.02)$ & $15.47(0.02)$ & $15.70(0.02)$ \\
2345.257 & A & $15.320(0.02)$ & $15.02(0.02)$ & $15.18(0.02)$ \\
2350.350 & B & $13.945(0.03)$ & $13.67(0.04)$ & - \\
2365.354 & B & $13.757(0.03)$ & $13.62(0.04)$ & $13.96(0.04)$ \\
2367.344 & B & $13.931(0.03)$ & $13.91(0.04)$ & - \\
2371.313 & B & $14.131(0.03)$ & $14.05(0.04)$ & $14.10(0.04)$ \\
2380.308 & B & $14.469(0.06)$ & $14.09(0.09)$ & $14.02(0.09)$ \\
2381.323 & B & $14.557(0.06)$ & $14.22(0.10)$ & $14.13(0.11)$ \\
2388.311 & B & $14.967(0.07)$ & $14.51(0.11)$ & $14.27(0.13)$ \\
2396.313 & B & $15.314(0.09)$ & $15.07(0.11)$ & - \\
2434.406 & A & $16.405(0.05)$ & $16.26(0.07)$ & $16.19(0.11)$ \\
\hline
\end{tabular}

computed simultaneously for all light curves (see Vinkó et al. 2001 for the description of the fitting method). The VRI light curves of the SN with the resulted MLCS model curves are presented in Fig. 3. The two earliest points before the maximum could not be fitted (although they are presented in Fig. 3), because the fiducial curves do not cover the early ascending branch before -10 days. The last point measured in the $I$ band seems to be too bright. This point was, however, included in the fitted dataset, as the individual $I$ images are of a good quality.

The residuals of the fitting are shown in Fig. 4. All points could be fitted within $\pm 0.2 \mathrm{mag}$, the overall standard deviation is $\sigma=0$. 115 .

The minimum of $\chi^{2}$ was found at the following parameters. $T_{0}(B)=\mathrm{JD} 2452346.1 \pm 0.8$ (fiducial B-maximum), $E(B-V)=$ $0.24 \pm 0.02, \mu_{0}=32.46 \pm 0.06, \Delta=-0.14 \pm 0.04$. The given confidence intervals correspond to 1-dimensional $\chi^{2}$ tests of each individual parameter with a significance level of $90 \%$. 


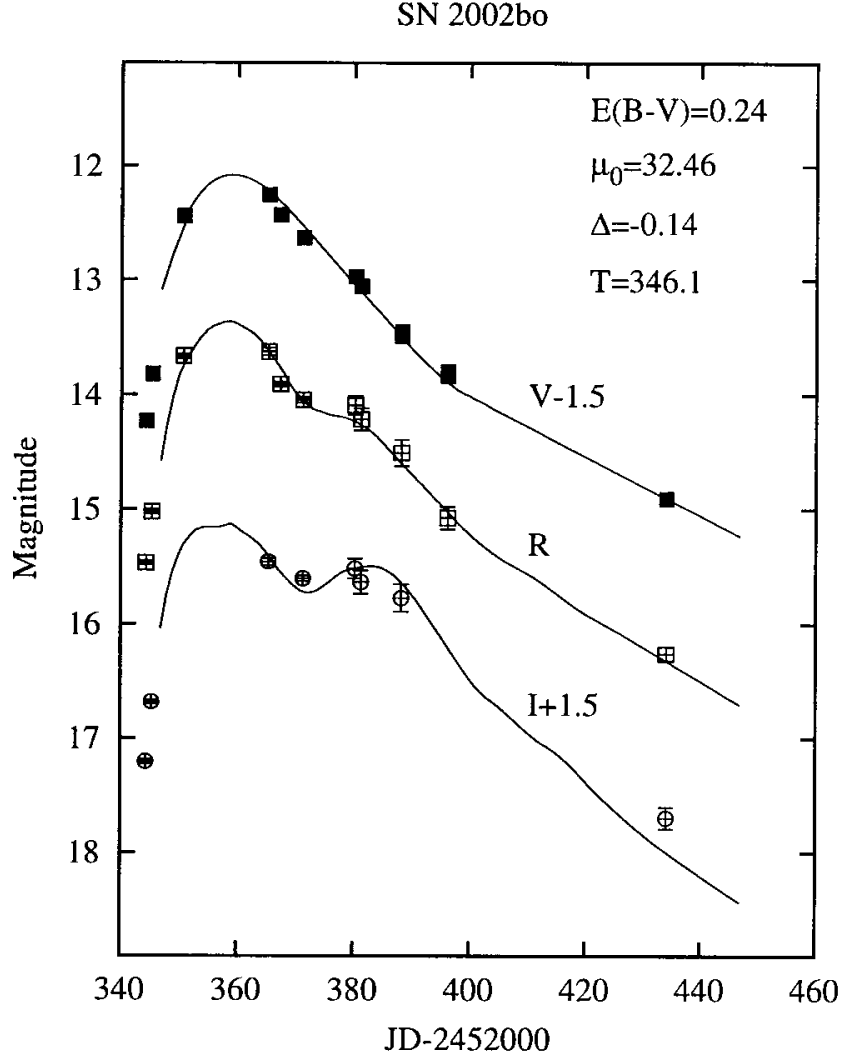

Fig. 3. Observed $V, R$ and $I$ light curves of SN 2002bo with the calculated MLCS light curve fit.

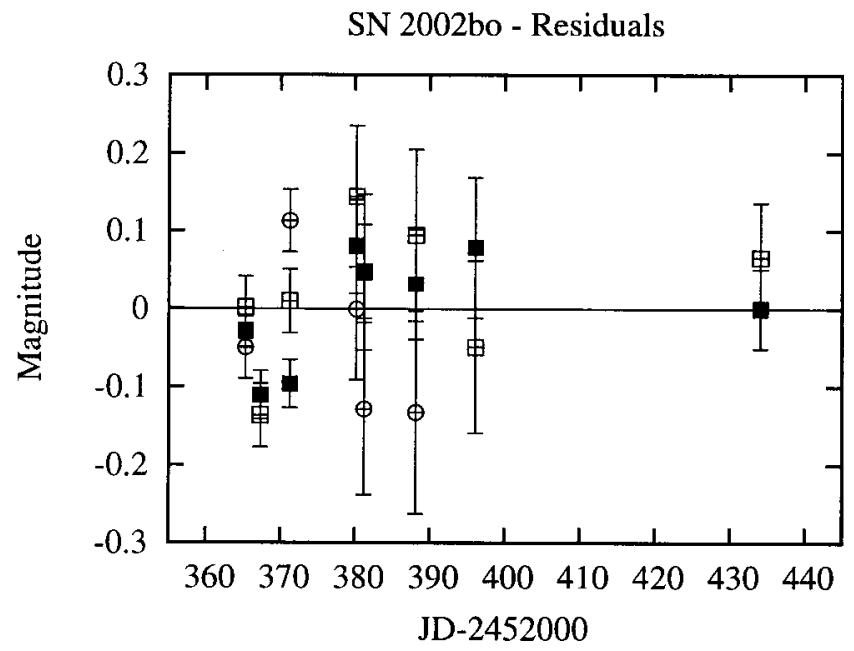

Fig. 4. Fitting residuals of the MLCS method. Symbols are the same as in Fig. 3.

The parameter $\Delta$ suggests a slight overluminous $\mathrm{SN}$. It is an interesting result, because early spectroscopy suggested a possible underluminous supernova (from the ratio of depths of the 5850 and $6355 \AA$ Si II lines, see Sect. 1). On the other hand, underluminosity would result in a precipitous decline rate. In our photometry, decline rates were found to be about normal or slightly steep, especially for the well-fitted $V$ and $R$ curves. The contradiction that early spectroscopy indicated an underluminous explosion, but later the photometry has shown a normal supernova, is an interesting subject for later studies.
GSC 142535

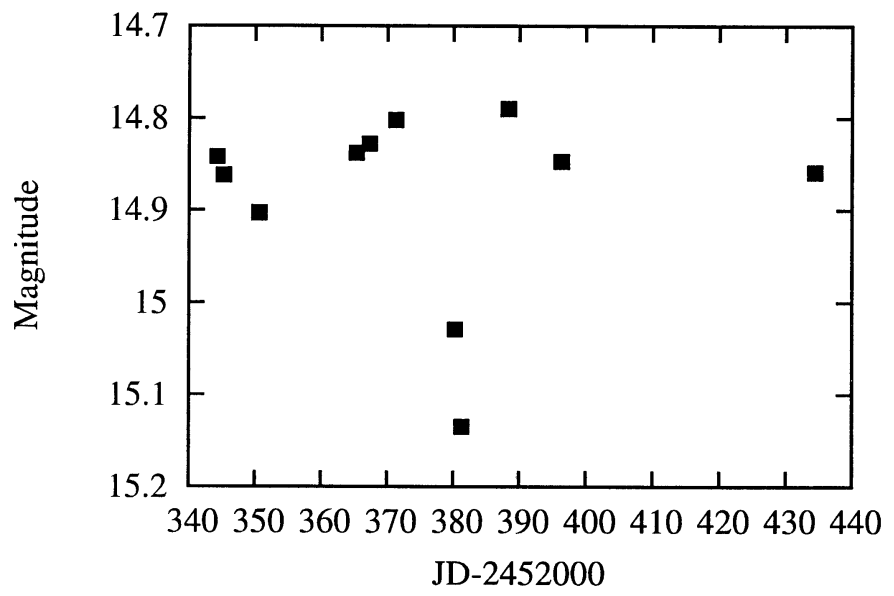

Fig. 5. $V$-band light curve of variable star C = GSC 142535 .

The MLCS analysis resulted in a new distance modulus to the host galaxy, $\mu_{0}=32.46 \pm 0.06$ mag (adopting $M_{V}=$ -19.46 for the fiducial SN curve). The real error of $\mu_{0}$ is probably increased by the errors of local standard magnitudes. Therefore, we suggest $\mu_{0}=32.46 \pm 0.10$ assuming that absolute magnitudes of local standards are measured more accurately than 0.08 .

Comparing the resulting color excess to the reddening due to Milky Way dust $(E(B-V)=0$ m $025 \mathrm{mag}$, Schlegel et al. 1998), it can be seen that the host galaxy ISM highly reddens SN 2002bo. This result is concordant with the spectroscopic results detailed in Sect. 1. Adopting $E(B-V)=0.24 \mathrm{mag}$, the total absorption in $V, R$ and $I$ bands is $A_{V}=0.77, A_{R}=0.92$, $A_{I}=0.43$ using the reddening law given by Schlegel et al. (1998).

\subsection{Variable star in the NGC 3190 field}

While selecting the appropriate comparison stars, differential magnitudes of the candidates (A, B, C, D, E stars in Fig. 1) were calculated. All stars showed constant light curves, but star C (GSC 1425 35) seemed to vary. This star was excluded from the comparison star set, and its light variation was measured with respect to stars A, B, D and E.

The undersampled $V$-light curve (Fig. 5) of star $\mathrm{C}$ shows at least 0.35 mag variation with a narrow primary minimum at about JD 2452379 and probable secondary minimum at about JD $2452356 \pm 6$. The general appearance suggests an eclipsing variable. The continuous variation might be due to $\beta$ Lyrae type, but a longer period may result in an Algol type light curve with considerable reflection effect. Its color indexes on JD 2434.406 UT were $V-R=0.52(3), R-I=0.47(2)$, which are not typical for Algol variables. However, based on few selected data points, no firm conclusions can be drawn either on the variability type or the light curve characteristics. 

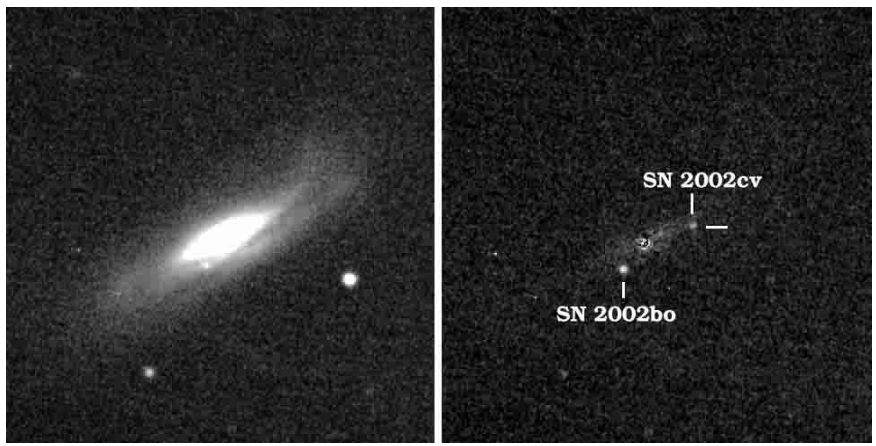

Fig. 6. I filtered observation of SN 2002bo and SN 2002cv on 8th June.

\subsection{Detection of $S N 2002 c v$}

The heavily obscured supernova SN 2002cv was discovered by Di Paola et al. (2003) on the 13th May. This is probably the most reddened supernova ever observed, its $A_{V}$ absorption is $7.8 \pm 1 \mathrm{~m} .0$.

We also detected this object on JD 2452434 (June 8, 2002), about 19 days after its maximum (Fig. 6). Despite the unfavourable sky conditions and high airmass, it is visible in the $I$-filtered image, taken with a total exposure time of $5 \mathrm{~min}$. The template subtraction allowed us to measure its brightness, which turned out to be $17.2 \pm 0.3$. This value is in a very good aggreement with the light curve taken by Di Paola et al. (2003). As far as we know, we have measured the last photometric point before the conjunction. Examining the $R$ and $V$ images, no firm evidence for the presence of SN $2002 \mathrm{cv}$ is found because of the high reddening.

\section{Conclusions}

1. VRI photometry of $\mathrm{SN} 2002$ bo is presented starting from 12 days before maximum light and extending up to 80 days past maximum. The maximum in the $B$ band occurred at about JD 2452346.1. In $V$ the brightness reached $13 \mathrm{~m} .58 \mathrm{mag}$ in maximum. Thanks to its detection in the $I$ band, a late photometric point is presented for $\mathrm{SN} 2002 \mathrm{cv}$ at JD $2452424.4, I=17.2 \pm 0.3$. A variable star is found in the field that is very close to host galaxy.

2. The reddening was estimated by applying the MLCS method for all light curves. This yielded $E(B-V)=$ 0 . 24(2) indicating significant extinction in host galaxy, while absorption caused by the the host galaxy is $A_{V}=$ 0 .77 using a standard reddening law. Spectroscopic observations confirms the presence of significant absorption by the ISM in NGC 3190.
The MLCS method assumes that the standard Galactic reddening law is valid in the SN host galaxy as well. It is worth noting that the observed VRI light curves could be fitted quite well when most of the reddening is due to the ISM in the host galaxy. This supports the reliability of the assumption made above.

3. The light curve analysis indicated that SN 2002bo was a slightly overluminous $\mathrm{SN}$ by about $0 \mathrm{~m} 14$ relative to fiducial SN type Ia. This is supported by the shape and the decline rate of the light curves, but is in contradiction with early spectroscopic data, where stronger Si II absorption features may have indicated a slightly subluminous explosion.

4. The distance of SN 2002bo inferred from the MLCS method was found to be $31.0 \pm 3 \mathrm{Mpc}$. This distance is about $50 \%$ higher than early dynamical and Tully-Fisher distances, but agrees fairly well with the most recent SBF determinations. These findings are concordant with earlier results that SBF and SN Ia distances both support the long extragalactic distance scale (de Vaucouleurs 1983; Ajhar et al. 2001).

Acknowledgements. This work has been supported by the Hungarian OTKA Grants T034615 and FKFP Grant 0010/2001. The kind hospitality of the Konkoly Observatory and their provision of telescope time is gratefully acknowledged. We thank our referee T. Matheson for his mindful reading and suggestions.

\section{References}

Ajhar, E. A., Tonry, J. L., Blakeslee, J. P., et al. 2001, ApJ, 559, 584 Benetti, S., Altavilla, G., Pastorello, A., et al. 2002, IAUC, 7848 Chornock, R., Li, W. D., \& Filippenko, A. V. 2002, IAUC, 7851 Di Paola, A., Larionov, V., Arkharov, A., et al. 2002, A\&A, 393, L21 van Driel, W., Marcum, P., \& Gallagher, J. S. 2001, A\&A, 378, 370 Kawakita, H., Kinugasa, K., Ayani, K., et al. 2002, IAUC, 7848 Landolt, A. U. 1992, AJ, 104, 340

Matheson, T., Jha, S., Challis, P., et al. 2002, IAUC, 7849

Héraudeau, Ph., \& Simien, F. 1998, A\&AS, 133, 317

Riess, A. G., Press, W. H., \& Kirshner, R. P. 1996, ApJ, 473, 88

Riess, A. G., Filippenko, A. V., Challis, P., et al. 1998, AJ, 116, 1009 Sárneczky, K., \& Bebesi, Zs. 2002, IAUC, 7863

Schlegel, D. J., Finkbeiner, D. P., \& Davis, M. 1998, ApJ, 500, 525

Tonry, J. L., Dressler, A., Blakeslee, J. P., et al. 2001, ApJ, 546, 681

de Vaucouleurs, G. 1983, ApJ, 268, 468

de Vaucouleurs, G., de Vaucouleurs, H., \& Corwin, H. G. 1991, Third Reference Catalog of Bright Galaxies (New York: Springer)

Vinkó, J., Bíró, I. B., Csák, B., et al. 2003, A\&A, 397, 115

Vinkó, J., Csák, B., Csizmadia, Sz., et al. 2001, A\&A, 372, 824 